\section{Cahiers de Narratologie}

Analyse et théorie narratives

31 | 2016

Sérialité narrative. Enjeux esthétiques et économiques

\title{
Entretien avec Françoise Lavocat. À propos de Fait et fiction. Pour une frontière, Paris, Seuil, «Poétique », 2016
}

Propos recueillis par Frank Wagner

Frank Wagner

\section{OpenEdition}

\section{Journals}

Electronic version

URL: http://journals.openedition.org/narratologie/7619

DOI: 10.4000/narratologie.7619

ISSN: $1765-307 X$

Publisher

LIRCES

\section{Electronic reference}

Frank Wagner, «Entretien avec Françoise Lavocat. À propos de Fait et fiction. Pour une frontière, Paris, Seuil, « Poétique », 2016 », Cahiers de Narratologie [Online], 31 | 2016, Online since 22 December 2016, connection on 14 November 2019. URL : http://journals.openedition.org/narratologie/7619 ; DOI : 10.4000/narratologie.7619

This text was automatically generated on 14 November 2019.

Article L.111-1 du Code de la propriété intellectuelle. 


\section{Entretien avec Françoise Lavocat. À propos de Fait et fiction. Pour une frontière, Paris, Seuil, « Poétique ", 2016}

Propos recueillis par Frank Wagner

Frank Wagner

1 Frank Wagner : Françoise Lavocat, à la lecture de votre dernier ouvrage en date, ce qui frappe de prime abord est l'ambition d'une telle « défense et illustration de la frontière entre fait et fiction", comme l'amplitude des recherches qu'elle a très certainement impliquées. Avant que nous n'entrions dans les détails, pourriez-vous commencer par présenter sommairement la genèse de cette réflexion remarquablement informée?

Françoise Lavocat: L'origine de mon intérêt pour la fiction est très ancienne. Dès ma thèse, sur le roman pastoral (publiée en 1997), j'ai été conduite à envisager la fiction comme monde - l'Arcadie «patrie mentale» depuis Virgile, selon Bruno Snell, ayant joué le rôle, pendant plusieurs siècles, de pays de la fiction par excellence. Mon deuxième ouvrage, sur Pan et les satyres (paru en 2005), m'a fait enquêter sur la mobilité du statut d'entités fictionnelles telles que ces créatures issues de la mythologie, mais qui, à partir du seizième siècle, oscillent entre réalité et fiction. Mais je me situe surtout dans la continuité du renouveau des études sur la fiction, dont les jalons marquants, pour moi, ont été les ouvrages de Thomas Pavel (Univers de la fiction, 1988), et Jean-Marie Schaeffer (Pourquoi la fiction, 1999). Pendant une dizaine d'années, j'ai approfondi ma réflexion sur la fiction, grâce à une réflexion collective, en confrontant les concepts contemporains et les textes anciens (Usages et théories de la fiction, les outils contemporains à l'épreuve des textes anciens, 2004), en analysant les apports de la théorie des mondes possibles aux études littéraires (La Théorie littéraire des mondes possibles, 2010), en enquêtant sur les conceptions de la fiction dans différentes aires culturelles (avec Anne Duprat, Fiction et cultures, 2010). 
3 FW : Réfléchir à l'existence de la frontière entre factuel et fictionnel présuppose une définition préalable des deux champs concernés. Votre ouvrage apporte certes une réponse en acte à cette question, mais vous serait-il possible, pour les besoins de cet entretien, de proposer une définition synthétique de ce qu'est pour vous la fiction?

FL: Les fictions sont des artefacts culturels fondamentalement non référentiels, qui contiennent néanmoins, la plupart du temps, des éléments qui réfèrent au monde réel.

On peut aussi définir les fictions comme des monde possibles impossibles (dans la mesure où ils reposent sur des paradoxes) où peuvent cohabiter des entités hétérogènes à deux titres: les personnages peuvent appartenir à une pluralité d'espèces (animaux parlants, anges, robots...) et avoir pour statut d'être fictionnels, transfictionnels (c'est-à-dire de provenir d'autres fictions), allégoriques, référentiels.

FW : Vous montrez fort bien que, pour des raisons diverses, et à partir de présupposés variés, des auteurs en apparence aussi éloignés que Lacan, Sollers, Forest, etc., procèdent à une forme de dissolution de la notion de fiction, à la (dé)faveur d'un curieux effet de syllepse, où le même terme ("fiction ") est pris dans deux acceptions substantiellement différentes. Pourriez-vous revenir sur ce gauchissement définitionnel, comme sur les conséquences - à vos yeux fâcheuses, si je vous ai bien lue - qu'il entraîne ?

FL: Les courants de pensée des années 1960-1970 ont en commun de ne pas s'être intéressés à la fiction, et même de lui avoir été fondamentalement hostiles. En ce qui concerne Lacan, je me suis surtout penchée sur la postérité de la déclaration selon laquelle "le réel, c'est l'impossible", qui a, selon moi, contribué à promouvoir un panfictionnalisme faisant disparaître la notion même de fiction: si tout est fiction, quelle place reste-t-il aux mondes imaginaires projetés par certains artefacts culturels? J'ai aussi voulu montrer qu'une certaine tradition intellectuelle et littéraire française, de Philippe Sollers à Philippe Forest, exprimait la conviction que tout était fiction tout en manifestant une grande agressivité à l'égard de la fiction, en particulier narrative, immersive, ou liée au spectacle : en d'autres termes la fiction cinématographique ou littéraire appréciée par un public large. J'ai essayé de sonder la généalogie et les conséquences de cet apparent paradoxe d'un rejet de la fiction, solidaire de l'idée selon laquelle tout (le sujet et le monde) était fiction. Cette position joue en effet sur plusieurs sens du mot « fiction ».

8 FW : Vous précisez dès votre "Introduction" (p. 12) qu'en termes méthodologiques, votre approche combine les angles sémantique, ontologique et pragmatique. Vous serait-il possible d'élucider les raisons et les implications de ce parti pris de diversification?

FL : Le renouveau des théories de la fiction, dans la lignée de Searle, et en France, de Jean-Marie Schaeffer, a jusqu'ici privilégié une perspective pragmatique (c'est aussi l'angle adopté, par exemple, par Olivier Caïra, qui a utilement étendu la réflexion sur la fiction aux jeux de rôles et aux jeux vidéo). Il est en effet indispensable, mais à mon avis insuffisant, de définir la fiction à travers ses usages : c'est ce que j'ai fait en proposant de distinguer la fiction du rite et du jeu. Quand j'ai identifié des environnements où la notion de fiction n'existait pas, en me fondant sur les travaux d'anthropologues comme Carlo Severi et Marika Moisseeff, je me suis intéressée aux aspects culturels des relations à l'imaginaire. Cependant une approche pragmatique, à mon avis, n'est pas 
exclusive d'une perspective ontologique, dont le domaine est celui des «théories qui tentent d'expliciter en quels types fondamentaux d'entités se distribue ce qui existe sous tel ou tel mode» (Pierre Livet). À partir du moment où je distingue des personnages fictionnels, historiques, allégoriques, que je m'intéresse au statut référentiel de tel ou tel monde, je pose ces questions en termes ontologiques. D'ailleurs, étant donné que la fiction et le concept de non-existant sont, logiquement et historiquement, étroitement liés, la perspective ontologique est à mon avis inséparable d'une réflexion sur la fictionnalité. Enfin, j'envisage aussi la fiction en termes de mondes possibles, dont le concept dérive de la sémantique kripkéenne, et dans cette perspective, j'accorde une grande importance aux paradoxes. Il n'y a aucune contradiction à examiner la fiction, ainsi que la différence entre fait et fiction, à partir de cette pluralité de points de vue, envisageant à la fois la relation de cette frontière à l'histoire, aux sociétés, au droit, et de façon conceptuelle, reposant sur la référence et la logique des mondes possibles.

FW : Nos échanges paraissent dans les Cahiers de narratologie. Or certains narratologues (Gérard Genette et Dorrit Cohn, en particulier) ont également tenté, au début des années 90 , de formaliser les frontières de la fiction. Comment vous situez-vous par rapport à leurs entreprises, comme par rapport à leurs conclusions?

11 FL : Je distinguerai de ceux de Gérard Genette les travaux de Käte Hamburger et de Dorrit Cohn qui ont au moyen d'une analyse logico-linguistique identifié des indices de fictionnalité internes aux textes. Ces résultats (poursuivis par les travaux d'autres chercheurs) restent parfaitement valables. Ils ont cependant été impuissants à contrebalancer la vague panfictionnaliste, sans doute parce que le périmètre de la fiction retenu par les deux narratologues (la fiction narrative en prose) était très étroit. Dorrit Cohn résiste même explicitement, au début du Propre de la Fiction (2001 pour la traduction française), à rapprocher la fiction de la notion d'imaginaire. Ne pas prendre en compte d'autres médias que le texte, aujourd'hui, paraît mal adapté à un examen de la notion de fictionnalité. En ce qui concerne Gérard Genette, il fonde essentiellement la distinction entre fait et fiction sur celle entre le narrateur et l'auteur, et j'ai essayé de montrer que cette position était fragile - elle a même, à mon avis, fragilisé la position différentialiste. Cependant, Gérard Genette a su, contrairement à Käte Hamburger et Dorrit Cohn, poser la question de la fiction dans le contexte intermédial contemporain. La place qu'il fait au cinéma, dans son dernier volume sur la métalepse (Métalepse, de la figure à la fiction, 2004) en témoigne. Ce faisant, il s'inscrit parfaitement dans le débat contemporain, et ma réflexion lui doit beaucoup.

12 FW : Autre question "de circonstances", puisque cet entretien paraît dans un volume consacré à Umberto Eco. Pour peu que vous estimiez avoir une « dette " à son égard, de quel ordre et de quelle ampleur est-elle exactement ?

13 FL : Umberto Eco a contribué de façon essentielle à la théorie littéraire des mondes possibles et ce, dès 1979. Par ailleurs, nombre de ses analyses, concernant, notamment, l'interprétation, ont nourri ma réflexion.

14 FW: Au titre des confusions somme toute fréquemment opérées par les théoriciens figure, je crois, l'assimilation problématique des notions de fiction, narration et littérarité. Pourriez-vous préciser comment, pour votre part, vous concevez leur articulation? 
FL : L'assimilation entre «fiction» et «narration » a plusieurs origines : d'une part, la confusion est favorisée par le sens du mot «fiction» en anglais, où il équivaut à "roman ». D'autre part, la domination de la littérature dans la littérature critique, et ce, dans les beaux jours du structuralisme et jusqu'à la fin du dernier millénaire, a pu entraîner une assimilation entre les trois termes cités. Il est pourtant bien évident que le phénomène de la fiction ne se limite en aucune façon à la narration en prose ni à la littérature. La fiction n'est pas davantage une qualité esthétique (les rêveries, les histoires que se racontent les enfants, maintes productions médiatiques ne prétendent pas à cette qualité). Gérard Genette, dans Fiction et Diction (1991), sépare d'ailleurs de façon nette fictionnalité et littérarité.

FW : L'un des aspects les plus originaux de votre recherche, peu usuel en matière de théorie de la fiction, réside dans l'étendue du spectre historique et géographique que vous parcourez. Sur ce double plan, à quel point les fluctuations de la notion de fiction et de ses usages vous paraissent-elles remarquables ?

FL : L'intérêt de déplacer l'enquête sur la fiction au-delà des frontières de l'Europe et des limites des $19^{\mathrm{e}}$ et $20^{\mathrm{e}}$ siècles m'est apparu depuis longtemps. En témoignent Usages et théories de la fiction, en 2004 et Fiction et cultures en 2010, qui font une large place aux siècles anciens, et, pour le second ouvrage collectif, aux aires culturelles les plus éloignées. Sur le plan chronologique, j'ai privilégié dans ce livre la confrontation entre les $16^{\mathrm{e}}-17^{\mathrm{e}}$ siècles et les $20^{\mathrm{e}}-21^{\mathrm{e}}$ siècles pour plusieurs raisons, dont, naturellement mes intérêts et mes compétences. Ce choix permet de minorer la place ordinairement réservée à la littérature romanesque du $19^{\mathrm{e}}$ siècle, et de mettre l'accent sur des caractéristiques de la fiction qui disparaissent pendant l'apogée du roman réaliste : les paradoxes et la pluralité ontologique des personnages. En outre, les conceptions de la fiction, au $17^{\mathrm{e}}$ et au $20^{\mathrm{e}}$ siècle, ne sont pas étrangères l'une à l'autre. J'ai trouvé par exemple des échos au scepticisme contemporain à l'égard de l'histoire (celui d'un Hayden White par exemple) dans celui d'auteurs comme La Mothe Le Vayer. De façon générale, la prise en compte de la longue durée m'a fait constater plus de permanences que de ruptures. Je m'oppose aux études, qui, reposant sur une conception trop étroite de la fiction, en assignent la naissance tantôt dans l'Antiquité, tantôt au Moyen âge, tantôt pendant la Renaissance, voire au $18^{\mathrm{e}}$ ou même au $19^{\mathrm{e}}$ siècle. Je défends l'idée d'une histoire longue et mouvementée de la fiction, fréquemment sujette à des essors qui rencontrent immanquablement des hostilités (jusqu'à aujourd'hui), ce qui entraîne parfois des périodes de déclin. Cependant, même si je m'inscris en faux contre ceux qui croient que le $20^{\mathrm{e}}$ siècle a tout inventé, mon attitude ne consiste pas non plus à considérer que tout a toujours déjà été dit. J'ai au contraire essayé de mesurer avec une certaine précision les différences entre les siècles anciens et la période contemporaine, à propos, en particulier, des métalepses et des voyages dans le temps.

18 De même, l'élargissement géographique de ma perspective, qui s'est notamment fait en faveur du Japon, m’a confortée dans l'idée que la fiction était une notion (et pas seulement une compétence et un usage), non seulement transhistorique, mais (presque) universelle. J'ai montré quelle conception sophistiquée de la fiction était explicitement exprimée dans le célèbre roman japonais de l'an mille de notre ère, le Dit $d u$ Genji. Par ailleurs, le Japon contemporain, avec des sociologues (comme Masachi Osawa) qui théorisent le fait que nous serions entrés dans une période post-fiction, m'a aussi paru intéressant, et j'ai essayé de rendre compte de ce type de pensée propre au contexte japonais. 
19 FW : Vous vous déclarez favorable à un "différentialisme modéré » (p. 12), et affirmez, entre autres, la nécessité politique des frontières de la fiction. Pourriezvous nous donner quelque idée des domaines dans lesquels cette dimension politique se fait jour?

20 FL : Ma défense d'un "différentialisme modéré » repose sur le fait que je définis, comme je l'ai dit précédemment, la fiction comme fondamentalement hybride, susceptible de contenir des éléments référentiels, qui sont d'ailleurs souvent responsables des conflits, des polémiques, et même des sanctions juridiques auxquelles sont souvent confrontés leurs auteurs.

21 En ce qui concerne les implications politiques de la frontière, j'ai argué que l'absence d'accord sur la différence entre les faits, en particulier historiques, et la fiction pouvait être source de manipulations. L'usage de la fiction, dans certains cas (j'ai cité celui de Jan Karski de Yannick Haenel) revient à présenter comme vraisemblables - et donc à donner à croire à - des versions des faits contraires à celles reçues par les historiens, sans produire l'ombre d'une preuve documentaire. Je sais très bien qu'aux Etats-Unis, des versions fictionnelles de l'histoire ont souvent été favorablement présentées, au contraire, comme la possibilité de contredire ou de compléter, de façon utile, l'histoire officielle, accusée de ne pas donner à entendre la voix des femmes, des minorités, des vaincus. Comme je suis hostile à une conception de la vérité comme ce qui serait politiquement ou moralement utile (on reconnaît là une des propositions de Richard Rorty), j'ai tendance à voir un danger dans le fait de confier à la fiction le rôle de dire contre les historiens - la vérité historique. Mais je ne m'attendais pas, en écrivant ce livre, à ce que cette problématique prenne une actualité brûlante : s'il est vrai que le mot de "post-vérité » est devenu le mot de l'année 2016, est-il pertinent de relier ce phénomène aux pensées qui, dans les dernières décennies du $20^{\mathrm{e}}$ siècle, ont battu en brèche l'idée d'une différence entre fait et fiction ? Il serait absurde de faire de Richard Rorty, de Hayden White ou des apôtres du storytelling les lointains responsables de la stratégie mensongère de Donald Trump, des pratiques de diffusion massive de fausses nouvelles à travers les réseaux sociaux, de l'indifférence d'une grande partie de la population à l'égard de la teneur de vérité des discours politiques. Cependant, il assez piquant pour moi, qui défends l'idée, parfois jugée conservatrice, selon laquelle est vrai ce qui correspond à des faits, de lire qu'une porte-parole de Trump (S. N. Hugues) déclare « qu'il n'y a pas vraiment de faits » (il n'y a que des opinions subjectives). L'idée que toutes les versions des faits se valent, parce qu'elles sont toutes construites, n'est plus l'apanage de pensées contestataires de gauche, bien au contraire. Je constate aussi que les médias qui tentent de s'opposer au populisme rivalisent désormais dans leur tentative d'opposer aux discours politiques la pratique du "fact checking ». Malgré le caractère largement répondu d'un constructivisme débridé, l'actualité politique montre les dangers de l'instrumentalisation de celui-ci.

FW : Nombre de littéraires, peu familiers de tels artefacts, ont pu être surpris voire choqués, à la lecture de Pourquoi la fiction? (1999) de Jean-Marie Schaeffer, de constater qu'il classait certains jeux vidéo au nombre des fictions. Sur ce point, vous vous livrez à un réajustement qui peut paraître très stimulant. Pourriezvous dès lors préciser ce qu'est, à vos yeux, la nature de la relation qu'entretiennent jeux vidéo et fiction?

FL : L'apparition de Lara Croft, à la fois sur la couverture d'un grand magazine féminin et dans un ouvrage de théorie littéraire, comme celui de Jean-Marie Schaeffer, a en 
effet fait date, produisant un effet de surprise, qui a séduit (c'est mon cas) et peut-être choqué. C'était en 1999. Depuis, le domaine de la ludologie s'est beaucoup développé, les jeux vidéo ont conquis une plus large part de l'audience planétaire, et plusieurs chercheurs spécialistes ont associé une réflexion approfondie sur la fiction avec des recherches sur les jeux vidéo (notamment Marie-Laure Ryan; en France, Olivier Caïra et Anne Besson). En ce qui me concerne, je me suis demandé ce que devenait la différence entre fait et fiction dans un environnement numérique, que ce soit celui de la réalité virtuelle en laboratoire, celui des mondes sociaux en ligne (du type Second Life) et celui des jeux vidéo. J'ai considéré que les environnements de synthèse présentaient une hybridité entre réalité et fiction tout à fait particulière, dans la mesure où intervenaient, d'une part, l'interactivité, et d'autre part, dans le cas des jeux, des règles. Par conséquent, j'ai proposé de distinguer jeu et fiction, parce que le jeu est inséparable de l'action, ce qui n'est pas le cas de la fiction. En outre, la relation au réel de la fiction se fait au moyen de la référence, tandis que dans le cas des univers de synthèse, elle passe par le geste et l'interactivité. Enfin, du point de vue des modalités (en particulier axiologique et déontique), celles-ci sont distribuées de façon tout à fait différente dans les fictions littéraires ou cinématographiques et dans les jeux vidéo: ceux-ci ne sont pas (ou en tout cas, ni principalement ni constitutivement), des mondes de "normes et de biens", pour reprendre une définition de Thomas Pavel, mais des mondes définis par des règles.

FW : Les neurosciences sont également, pour la plupart des littéraires, terra incognita. D'après les travaux des spécialistes que vous avez exploités, peut-on vraiment affirmer que nous possédons un " équipement cognitif " susceptible de nous permettre de distinguer avec assurance factuel et fictionnel ? En effet, la fragilité de la mémoire d'une part, la conception même des expériences supposées permettre d'en juger d'autre part, n'incitent-elles pas à la prudence en la matière?

FL : Les résultats des expériences en neurosciences sont en effet à manier par les non spécialistes (qui n'ont d'ailleurs pas accès à ces expériences elles-mêmes) avec la plus grande prudence. J'ai moi-même montré quelle utilisation sauvage avait été parfois faite de la découverte des neurones miroir, dans les années 1990, pour arguer de l'indistinction entre réalité et imaginaire (puisque certaines zones du cerveau, dévolues à l'imitation et la simulation perceptive, réagissent de la même façon devant un mouvement effectué par une personne en présence que devant un mouvement représenté, filmé par exemple). Pourtant, il existe beaucoup d'expériences, à partir des années 2000, concernant les tâches de contrôle de la réalité, qui montrent que le cerveau humain est effectivement équipé pour distinguer les faits imaginaires et la perception d'événements réels: les défaillances, dans ce domaine, peuvent être symptômes de psychose (il y a cependant des sociétés traditionnelles, comme je le montre dans le livre en m'appuyant sur des travaux d'anthropologues, où ce partage est inexistant ou bien différent de celui qui prévaut dans les sociétés occidentales contemporaines). En ce qui concerne les réactions cognitives à la perception de personnages historiques et fictionnels, qui requiert des compétences culturelles, c'est évidemment plus délicat: les seules expériences menées dans le domaine des neurosciences concernant cette question spécifique, à ma connaissance, sont celles d'Anna Abraham et de son équipe (en 2009 et 2010), qui a montré que les sujets, face à des scenarii impliquant un personnage fictionnel (Cendrillon) et réel (George Bush) activaient différents types de mémoire (sémantique ou épisodique). Cependant, en 
effet, la mémoire étant l'élément le plus fragile de notre équipage cognitif, ce genre de distinction peut s'altérer, de même que les sujets sains sont susceptibles de produire, très facilement, de faux souvenirs.

FW : De longue date, la relation du lecteur et/ou du spectateur à la fiction a été conçue en termes d'identification - dont le principal support serait le personnage. Or les sciences cognitives, que vous mettez à contribution au cours de votre enquête, incitent semble-t-il à se montrer circonspect au moment de raisonner en ces termes. Pourriez-vous donc préciser en quoi une analyse de la réception des fictions à l'aune de tels présupposés "identificatoires " peut paraître sujette à caution?

FL: Il n'est nul besoin de faire appel aux sciences cognitives pour faire le constat suivant: au spectacle de Guignol, les enfants ne se prennent pas pour Guignol. En revanche, ils avertissent volontiers, et bruyamment, Guignol de l'arrivée du gendarme. Les fanfictions contemporaines témoignent du désir largement partagé de pénétrer dans l'univers fictionnel, de se mêler, sous la forme d'un personnage, à la société des héros de l'histoire. Dans ces réécritures, les lecteurs ne veulent pas prendre la place de leur héros favori : ils ambitionnent plutôt de frayer en sa compagnie, de lui prêter mainforte, de lui servir d'adjuvant. Ces constat m'ont amenée à privilégier l'empathie sur l'identification, même si je ne nie pas que certaines fictions puissent susciter des pratiques qui y ont trait : pourquoi, sinon, des hommes du $17^{e}$ et du $18^{e}$ siècle auraientils signé leurs lettres tendres du nom de "Céladon »? Pourquoi tant de jeunes gens de par le monde aiment-ils coiffer le casque et se draper dans la cape de Darth Vader? Cependant, l'empathie (dont le fonctionnement a beaucoup été éclairé par la psychologie et les neurosciences), me semble marquer de façon constitutive le rapport à la fiction. L'empathie est à mon avis à l'origine d'un mouvement contrarié. Face à une fiction, l'impulsion à agir, à venir en aide à notre prochain, est obligatoirement suspendue. Cette frustration me paraît centrale dans le rapport à la fiction. Elle éclaire aussi certaines attitudes à l'égard de la frontière entre fait et fiction, que tant de lecteurs ou de spectateurs brûlent de traverser et que tant de théoriciens croient avoir renversée.

FW : Vous avez antérieurement publié un ouvrage intitulé La Théorie littéraire des mondes possibles (2010). Or il paraît probable qu'une réflexion sur les frontières de la fiction ne puisse faire l'économie, au moins ponctuellement, d'une perspective ontologique, c'est-à-dire centrée sur le "mode d'être " des entités fictionnelles comme des univers qu'elles peuplent. Toutefois, puisqu'il n'a pas manqué de théoriciens pour faire preuve de réticences à ce propos, pourriez-vous nous dire en quoi la théorie des mondes possibles peut permettre de penser les frontières de la fiction?

FL : La théorie des mondes possibles suscite en effet souvent des réticences, en raison des difficultés qu'elle présente, dues à son origine logico-mathématique, et des inévitables déplacements et simplifications auxquelles procèdent les théories littéraires qui s'en inspirent. Dès 1988, Thomas Pavel recommandait de faire de la notion de " monde possible » un usage " distant». La prudence est d'autant plus de mise que les notions de "monde » et de "possible » sonnent de façon faussement familière aux oreilles des littéraires: le risque est grand de les réduire à des banalités. Enfin, si la perspective ontologique rebute certains, c'est parce qu'ils lui opposent une approche pragmatique : comme je l'ai dit précédemment, la fiction peut et doit être abordée 
selon l'une et l'autre perspective. L'ontologie est à tort associée au dogmatisme, à l'essentialisme, à la métaphysique. Pourtant, à partir du moment où l'on se demande quelle est la nature d'un monde et d'un personnage fictionnel, où l'on réfléchit au paradoxe que présente l'existence d'entités non existantes, on pose des questions de nature ontologique.

L'intérêt que présente à mes yeux la notion de monde possible est qu'elle permet d'envisager les fictions sous la forme de constellations de variantes, d'adaptations, de réécritures et de suites. Elle permet de poser des questions nouvelles: celle de la constitution de ces mondes en termes de modalités logiques (c'est ce qui m'a permis d'éclairer la distinction entre les fictions traditionnelles et les jeux vidéo) ; celle des relations entre ces mondes (compris comme des collections d'états de choses); celle des modalités de passage d'un monde à l'autre. Dans cette optique, l'accent est mis sur la transfictionnalité (le passage pour un personnage d'un monde à l'autre), les contrefactuels, les métalepses. Envisager les fictions comme des mondes, leurs constellations d'imitations, de brouillons, d'adaptations, de traductions, comme des univers implique des différenciations entre ces artefacts, et donc, de façon métaphorique, des frontières. Pour apprécier les passages d'un monde à l'autre (c'est le cas de la transfictionnalité, de la métalepse), il faut bien que l'univers de départ et celui d'arrivée soient distincts; il faut bien, d'un point de vue conceptuel, qu'ils aient des frontières.

31 FW : Cette question en entraîne une autre. Dans la dernière partie de votre ouvrage, vous repérez en effet plusieurs "paradoxes de la fiction ". Vous serait-il possible de rappeler brièvement en quoi ils consistent, comme la façon dont les lecteurs/spectateurs paraissent réussir à s'en accommoder en les « naturalisant "?

32 FL : La remarque selon laquelle les fictions ne seraient pas des mondes possibles parce qu'elles ne sont pas entièrement consistantes a été souvent faite (par Thomas Pavel par exemple). J'ai argué que les paradoxes étaient une des caractéristiques propre des fictions (le monde réel n'étant pas constitué de propositions, il n'est pas sujet à être sémantiquement paradoxal) et que l'on peut donc les définir comme des «mondes possibles impossibles». Les fictions reposent fondamentalement sur le paradoxe de l'existence du non existant, souvent thématisé dans les fictions mêmes. Par ailleurs, les fictions abondent en impossibilités qui passent la plupart du temps inaperçues (Olivier Caïra en a fait la liste dans Définir la fiction, en 2011). J'ai pour ma part proposé de distinguer les paradoxes structurants de nombreuses fictions, et qui déclinent celui du menteur ou relèvent de l'inclusion réciproque. Je me suis intéressée aussi à certains paradoxes thématisés dans les fictions, comme celui du voyage dans le temps. J'ai constaté, en analysant de nombreux blogs et commentaires en ligne de nouvelles de Borges ou de Cortázar, ou Mullholland Drive de David Lynch, que leurs paradoxes déclenchaient chez les lecteurs ou les spectateurs une intense activité herméneutique, visant à rationaliser, réduire ces paradoxes. Je me suis aussi appuyée sur des études en psychologie et en neurosciences qui montrent que les paradoxes et les contradictions génèrent une activité cognitive spécifique, dédiée à leur résolution. Ainsi, les paradoxes constitutifs de la fictionnalité soit passent inaperçus, soit, s'ils sont particulièrement visibles ou insistants, déclenchent une importante activité interprétative en vue de les résoudre. D'ailleurs, certains de ces paradoxes présentent un véritable danger pour le monde fictionnel, qu'ils peuvent détruire (c'est tout particulièrement le cas pour les 
paradoxes temporels à l'œuvre dans les voyages dans le temps). Ainsi, il y a une sorte de tension entre les paradoxes, facteurs de perturbation ou même de destruction, et la bienveillance herméneutique des lecteurs ou des spectateurs: celle-ci les engage à aménager le monde de fiction, le rendre habitable, en d'autres termes, à rendre possible l'immersion fictionnelle.

FW : Au cours de cette enquête, vous recensez nombre de fictions (littéraires, cinématographiques, télévisuelles...) dont la particularité majeure est de dialectiser la frontière entre fiction et réalité. Or cette activité de dialectisation emprunte une voie que l'on peut elle-même considérer comme paradoxale, celle de la métalepse - notion dont la récente fortune critique est indéniable. Vous serait-il possible de préciser en quoi la représentation d'une transgression de la frontière entre fiction et réalité vous paraît en définitive plutôt œuvrer à sa préservation, voire à son renforcement ?

$\mathrm{FL}$ : C'est vous qui employez le mot de « dialectiser » la frontière entre fait et fiction par la métalepse. Je me contenterai plus modestement du terme "problématiser", ou encore plus simplement, "mettre en évidence ». Il est en effet indéniable qu'un très grand nombre de fictions contemporaines, sous toutes les formes et sur tous les supports médiatiques qui existent, multiplient les métalepses ayant une portée métafictionnelle. Lorsque, par exemple, dans une petite série télévisée anglaise (Lost in Austen, en 2008) une jeune lectrice se retrouve dans le monde de Pride and Prejudice, prend la place de Lizzie Bennet (qui se retrouve dans le monde contemporain et s'en trouve bien), s'évertue en vain à orienter les événements pour les faire coïncider avec ceux du roman de Jane Austen, et finit par épouser Darcy, ce sont beaucoup de phénomènes liés à la lecture qui sont interrogés : le fragile prestige de l'auctorialité, la capacité des personnages de romans à traverser les époques (Lizzie Bennet est peutêtre le personnage à qui sont actuellement attribués le plus de comptes Facebook, le plus de blogs), le désir des lecteurs.trices pour les personnages... Le charme de l'œuvre réside dans le fait qu'elle rend possible ce qui ne l'est pas, y compris aux yeux du personnage principal, la lectrice. Lorsque celle-ci pince une des sœurs Bennet pour apprécier la consistance du corps d'un personnage fictionnel (question que ce genre de fantaisies métaleptiques pose systématiquement), elle rend manifeste le paradoxe du non existant, et par le biais de la simulation perceptive, nous invite à imaginer ce contact impossible. Le sourire que fait naître (sur les lèvres du spectateur ou de la spectatrice) le spectacle final, celui de la lectrice enlacée par l'objet du désir, le fictionnel Darcy, est à la mesure de la conscience de cette impossibilité. Cet exemple, et bien d'autres, me donnent à penser que le plaisir de la métalepse consiste précisément à nous faire voir (sous la forme d'armoires, de terriers, de miroirs, de tornades...), et même palper, mentalement, cette frontière.

FW : Pour un narratologue, ce qui peut paraître surprenant dans vos analyses tient au fait que vous analysez de façon privilégiée les "métalepses intrafictionnelles » (p. 497 sq.), et affirmez que «ce n'est qu'à l'intérieur de la fiction qu'il est possible de représenter la frontière entre réalité et fiction et de la traverser effectivement " (id.); au lieu que Genette, par exemple, présente comme plus troublantes les métalepses mettant aux prises les niveaux intra- et extradiégétiques. Comment justifier ce renversement de perspective? 
36 FL : Je ne crois pas du tout qu'il y ait un renversement. Ma position sur la métalepse est très proche de celle de Gérard Genette, et je ne m'oppose qu'à ceux qui banalisent à l'extrême le concept, en voyant de la métalepse partout.

J'ai simplement mis l'accent sur le fait que les métalepses contemporaines étaient beaucoup plus littérales que celles du passé (avant le fin du $19^{\mathrm{e}}$ siècle), et que les métalepses dites «rhétoriques", celles, par exemple, où un narrateur apostrophe le lecteur ou un de ses personnages, font bien pâle figure à côté de celles où narrateur, lecteur et personnages se retrouvent effectivement dans un même monde. C'est le cas, par exemple, dans Niebla de Miguel de Unamuno (1914), où le personnage finit par rencontrer son auteur, Miguel de Unamuno, qui déclare vouloir le tuer - de fait, il meurt. Il me semble que parler de niveaux intradiégétique et extradiégétique focalise l'attention sur le narrateur, et donc sur les métalepses rhétoriques, dont Monika Fludernik, par exemple, a montré la fonction de régie (2005). Ces métalepses sont de loin les moins troublantes. M'intéresser aux métalepses intrafictionnelles est la seule alternative, car je ne crois pas qu'il y jamais de pénétration réelle de la fiction dans le monde réel, et du monde réel dans la fiction (je laisse à part, ici les phénomènes liés à l'interactivité). Genette n'a jamais parlé du monde actuel faisant irruption dans le monde fictionnel, ou inversement, sauf, dans son dernier livre sur la métalepse (2004), à propos du cinéma, où il rapproche, à propos de la force de suggestion de l'image, la métalepse de l'hypotypose : mais dans ce cas, il ne s'agit pas du tout des effets les plus troublants de la métalepse, mais au contraire d'une conception très diluée de celle-ci. C'est cette banalisation de la métalepse, que certains critiques (Jean Bessière ou Yves Citton notamment) veulent confondre avec les effets de la fiction, que je refuse.

$\mathrm{Au}$ contraire, en totale conformité avec la définition traditionnelle de Genette, de la métalepse comme transgression, produisant une impression de bizarrerie, je m'intéresse à celles qui produisent cet effet de la façon la plus intense. C'est le privilège de l'époque contemporaine de pousser à l'extrémité les potentialités de cette figure. Pour reprendre l'exemple classique de Fontanier, le $17^{\mathrm{e}}$ siècle voyait une métalepse dans l'expression «Virgile fait mourir Didon ». Un auteur de la fin du $20^{\mathrm{e}}$ ou du $21^{\mathrm{e}}$ siècle représenterait Virgile allumant lui-même le bûcher.

Cette littéralité rend assez peu opérante, il est vrai, la distinction entre «niveau intradiégétique » et «extradiégétique ». Pour reprendre l'exemple précédent, Miguel de Unamuno, lorsqu'il rencontre, en tant que personnage, sa malheureuse créature, n'appartient pas à « un niveau extradiégétique » : il serait plus juste de dire que le nom propre produit un effet illusoire de présence réelle, et que le personnage Unamuno a une contrepartie dans le monde réel, qui est l'auteur. Lorsque se produit ce qu'on appelle un effet cameo, au cinéma, (quand Hitchcock en tant qu'Hitchcock fait une apparition dans ses films), il y a un véritable effet de présence réelle, mais il ne s'agit pas non plus d'un "niveau extradiégétique» qui empiéterait sur le niveau « intradiégétique ». En tout cas, je ne l'ai pas analysé de cette façon.

FW : Question corollaire de la précédente : au sein des fictions contemporaines, tous médias confondus, ne peut-on repérer aucun exemple de métalepse authentiquement dissolutive?

41 FL: Je n'en distingue en effet aucun. Mais je suis prête à réviser ma position si on m'en fournit un exemple convaincant. 
42 FW : En «Conclusion » (p. 522), vous déclarez plaider « en définitive, pour une appréhension de la fiction à travers ses différentes modalités d'hybridation avec le factuel, qui font, la plupart du temps, ressortir les contours de la fictionnalité et de la factualité plutôt qu'elles ne les effacent ». La question qui s'impose est proche de la précédente, mais excède largement le cas somme toute particulier de la métalepse - et semble en outre légitimée par la précision temporelle «la plupart du temps "... Innombrables sont en effet les textes récents qui s'ingénient à brouiller les frontières du fictionnel et du factuel. Ne pourrait-on dès lors estimer que cette prolifération, loin de renforcer la frontière, est tout de même susceptible de finir par la fragiliser?

FL : Il existe en effet des enclaves où la question du factuel et du fictionnel ne se pose pas, à tout le moins pour la majorité des lecteurs ou des spectateurs. C'est le cas de maintes autofictions (pourvu que le lecteur n'appartienne pas à l'entourage de l'auteur) ou d'émissions de divertissement. Je me rappelle ma propre perplexité, aux Etats-Unis, face à une émission de téléréalité de Jerry Springer, où un prêtre d'allure louche faisait irruption et mariait deux des protagonistes : étaient-ils vraiment mariés ou non? Ayant posé la question aux gens qui m'entouraient, je n'ai eu d'autre réponse que « on ne sait pas ", « peu m'importe ». Il y a aussi des textes littéraires qui parviennent à suspendre tout pacte de fictionnalité ou de factualité. Cependant, je constate aussi que les internautes qui découvrent, par exemple, qu'une confession autobiographique en ligne était en fait jouée par une actrice, expriment fréquemment des réactions outragées. J'ai aussi remarqué que des films ou des émissions où le partage de la fiction et de la réalité était malaisé (comme Curb your enthousiasm de Larry David), ou relevaient d'un montage sophistiqué (comme Adaptation de Spike Jonze), suscitaient beaucoup de commentaires, de pages Wikipedia, de blogs, dédiés au triage des briques fictionnelles et factuelles. Je ne sais pas si la répétition des mêmes dispositifs de brouillage, la multiplication des métalepses (qui en ont probablement usé les effets), ont abouti à un effacement effectif de la frontière. D'une part, l'augmentation très sensible des procès intentés aux auteurs et éditeurs de fiction (pour référentialité jugée abusive) suggère une réponse négative ; d'autre part, il est possible, si nous vivons bien à l'ère de la "post-vérité, » que la confusion entre faits, mensonges, storytelling, fictions se soit accrue. Mais comme je l'ai dit précédemment, je constate l'existence d'une réaction collective, aussi bien de la part de philosophes (je pense à Pascal Engel) que de journalistes contre cette confusion. FW : À la fin de votre ouvrage, vous affirmez que « Les fictions sont des mondes à interpréter, ce que n'est pas, ou pas de la même façon, le monde réel. " (P. 533). Pourriez-vous préciser en quoi consiste la différence entre les activités interprétatives suscitées par la fiction et le monde (non-fictionnel) ?

FL : On sait, depuis Schleiermacher, que l'interprétation est une activité cognitive qui touche tous les aspects de notre vie. Nous interprétons les paroles et les actes de nos semblables aussi bien que les artefacts culturels. La distinction que j'introduis dans ce passage défend plutôt l'idée selon laquelle les fictions réclament une démarche herméneutique spécifique. J'ai défendu l'idée selon laquelle les mondes de fiction étaient incomplets, tissés de contradictions, et que l'interprétation visait en quelque sorte à combler les manques, à résoudre les contradictions, afin de permettre l'immersion et la jouissance du monde fictionnel. C'est de cette façon que je comprends la « coopération » du lecteur selon Umberto Eco. 
FW : En guise de mot de la fin, vous témoignez d'un amour de la fiction qui paraît trouver à s'incarner idéalement dans le personnage, qui « est et sera toujours là quand auront disparu le roman et les livres " (p. 535). À l'issue de votre enquête, et par-delà la question des frontières, ne discernez-vous pas de singularités de la fiction littéraire, qui autoriseraient un rapport « amoureux » spécifique?

$\mathrm{FL}$ : C'est à propos d'un personnage très éloigné de tout univers littéraire, Di Gi Charat, ou Kyaratto, une mascotte publicitaire japonaise devenue à la fin des années 1990 un personnage de manga, d'anime, de jeux, support de multiples transformations, adaptations et imitations (y compris au niveau vestimentaire et comportemental des jeunes gens), et ce au niveau planétaire, que j'ai affirmé que le personnage survivrait au roman.

Même s'il y a lieu de distinguer un personnage littéraire (entièrement construit par le lecteur), filmique (inséparable de l'acteur qui l'incarne), théâtral (incarné par une série d'acteurs différents, en ce qui concerne en tout cas les pièces du répertoire), ils suscitent tous l'empathie, le désir empêché de leur venir en aide, de pénétrer dans leur monde. Je pense que le personnage transcende le média. Le culte et l'amour que les Japonais vouent, non seulement aux marionnettistes, mais aux marionnettes ellesmêmes (dans le cadre du bunraku, théâtre traditionnel), témoignent de ce fait. Les multiples incarnations des personnages contemporains, déclinées dans des mangas, des jeux, en figurines, cartes ou poupées le suggèrent aussi. On a cru que les avatars des mondes sociaux en ligne (du type Second Life), ou des jeux vidéo, renforceraient encore cette relation aux personnages (voire entraîneraient des phénomènes d'identification dangereux) car les internautes des années 1990 assuraient que leurs avatars étaient des prolongements d'eux-mêmes : mais la banalisation du média, l'habitude, ont créé la distance et le détachement des digital natives à l'égard de leurs avatars. Ce serait même plutôt dans cette indifférence que résiderait la distinction entre personnages fictionnels et avatars ludiques : l'avatar-personnage d'un jeu, aux multiples morts, peu enclin aux conflits moraux, sans autre destin que l'issue d'une partie, me parait susciter un intérêt bien différent de celui d'un personnage de fiction traditionnelle. Mais l'évolution des jeux, leur complexification, y compris en termes éthiques, est telle, que les ludologues affirment que cette distinction est vouée à disparaître. J'avoue que je n'en sais rien.

Tout ce que je puis dire, pour conclure, c'est que mes travaux sur la fiction ont été rendus possibles par le retour du personnage sur la scène critique, et l'autorisation qui a été donnée, à la fin des années 1980, par Thomas Pavel, puis, dix ans plus tard, par Jean-Marie Schaeffer, de les considérer comme autre chose que des êtres de papier ; en un mot, de les aimer. Il n'y a pas d'amour pour la fiction sans amour pour les personnages. 